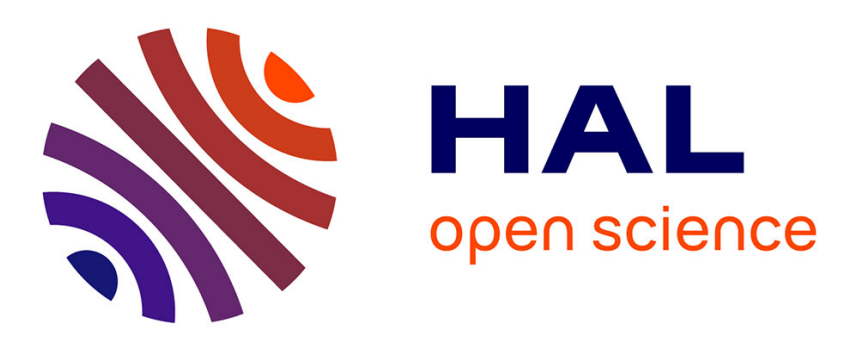

\title{
Hyper-reduced direct numerical simulation of voids in welded joints via image-based modeling
}

Laurent Lacourt, David Ryckelynck, Samuel Forest, Victor Rancourt, Sylvain Flouriot

\section{- To cite this version:}

Laurent Lacourt, David Ryckelynck, Samuel Forest, Victor Rancourt, Sylvain Flouriot. Hyper-reduced direct numerical simulation of voids in welded joints via image-based modeling. International Journal for Numerical Methods in Engineering, 2020, 10.1002/nme.6320 . hal-02502006

\section{HAL Id: hal-02502006 https://hal.science/hal-02502006}

Submitted on 8 Mar 2020

HAL is a multi-disciplinary open access archive for the deposit and dissemination of scientific research documents, whether they are published or not. The documents may come from teaching and research institutions in France or abroad, or from public or private research centers.
L'archive ouverte pluridisciplinaire HAL, est destinée au dépôt et à la diffusion de documents scientifiques de niveau recherche, publiés ou non, émanant des établissements d'enseignement et de recherche français ou étrangers, des laboratoires publics ou privés. 
DOI: $\mathrm{xxx} / \mathrm{xxxx}$

\title{
ARTICLE TYPE
}

\section{Hyperreduced direct-numerical-simulation of voids in welded joints via image-based modeling}

\author{
Laurent LACOURT ${ }^{* 1,2}$ | David RYCKELYNCK ${ }^{1}$ | Samuel FOREST ${ }^{1}$ | Victor de \\ RANCOURT $^{2}$ | Sylvain FLOURIOT ${ }^{2}$
}

\footnotetext{
${ }^{1}$ Centre des Matériaux (MAT), MINES ParisTech - PSL Research University CNRS UMR 7633, BP 8791003 Évry,

France

${ }^{2}$ Centre d'Études de Valduc, CEA, 21120

Is-sur-Tille, France

Correspondence

*Corresponding author : Laurent LACOURT

Email: laurent.lacourt@mines-paristech.fr

\section{Present Address}

This is sample for present address text this is sample for present address text
}

\begin{abstract}
Summary
Defects such as gas pores can be formed and trapped in the fusion zone during laser welding. These defects can affect significantly the mechanical reliability of the welded joint. Current non-destructive inspection technologies are able to detect micro-voids in a mass production context. Finite Element Analysis can therefore be used to assess the lifetime of an observed component via image-based modeling. Unfortunately, running a simulation per component entails a huge and generally unaffordable computational cost. In addition, voids do not admit a parametric modeling. In this paper, a numerical method is proposed to study the impact of defects on the mechanical response of a welded joint. It is based on model order reduction techniques that decrease the computational cost of each simulation related to an image-based modeling. To tackle the reduction of non parametric defects, a multi scale construction of the reduced basis is proposed, although no scale separation is assumed when computing the mechanical response of the structure. Some empirical modes are representing the structure behavior and other empirical modes are related to the defect-induced local fluctuations. They are then assembled to simulate a defective joint. Assets and limitations of the proposed method are explored through a simplified 2D problem. For the sake of reproducibility, this 2D problem is fully parametric. Finally, a realistic 3D industrial case is presented, where voids geometries have been measured via computed tomography. This 3D problem being non parametric, fluctuation modes must be computed on the fly, once the computed tomography has been performed.
\end{abstract}

\section{KEYWORDS:}

Reduced Order Model, Impossible sampling, Elasto-Plasticity, Material health monitoring, Combinatorial model order reduction

\section{1 | INTRODUCTION}

Direct numerical simulations (DNSs) have been introduced in fluid mechanics to account for the wide range of scales in turbulent flows [1], without using a simplified modeling of motions at small scales. In mechanics of heterogeneous materials, DNSs can be used as reference solutions for stresses and displacements in order to assess the accuracy of homogenization theories as proposed 
in [2, 3]. This can also help to develop physical models with a deep understanding of deformation mechanisms as in [4, 5, 6, 7]. In the present paper, a method dedicated to the direct numerical simulation of welded joints containing void defects is proposed. The numerical simulation aims at determining whether a defect will cause an early fatigue failure of the welded joint or not. Fatigue cracks generally initiate at these internal defects, acting as stress concentrators [8]. The need for such DNSs increases as non destructive inspection of serial produced components spreads in the manufacturing industries. Non destructive inspection techniques are able to detect and locate voids for a wide range of materials and welding processes: resistance seam welding of aluminium, zinc and galvanised steel [9], resistance spot welding of ferritic/martensitic steels [10], electron beam welding of steel to Fe-Al alloy [11] or laser welding of stainless steels [12] and aluminium alloys [13]. Moreover, image-based meshing methods [14] enable to generate complex finite element meshes of 3D digital images obtained by such techniques. Hence, with a convenient informatics integration platform, as proposed in [15], DNSs for defect modeling via finite element simulations can be achieved. Unfortunately, it cannot be used as a tool to assess the quality of a component in a serial production framework. The required fine meshes generally lead to prohibitive computational time particularly when cyclic loadings are considered. In recent years, model reduction methods have been developed for parametric problems, like the Proper Orthogonal Decomposition (POD) method [16, 17, 18] or the Proper Generalized Decomposition (PGD) method [19], as well as the hyper-reduction method (HRM) [20, 21]. These methods are used to decrease the computational cost of numerical simulations: balance equations are projected onto an empirical reduced basis in order to speed-up numerical predictions. Therefore, they are of huge interest when limited ressources are available.

Model order reduction techniques have already been applied to assess the effect of local modifications on structural failures. The static condensation reduced basis method [22] is used in [23] to solve Helmholtz partial differential equations in complex geometries. A library of reduced order models is built for subparts of the component. To simulate the full geometry, these reduced order models are assembled. In [24], the PGD is used to estimate the stresses within plates containing holes. However, such an approach cannot be applied in the current case because no parametrization can be achieved. Indeed, it is not realistic to think that the high spatial resolution of 3D digital images could be parametrized. Usual offline-online approaches developed for reducing parametric nonlinear mechanical problems are no more appropriate here. The ArbiLoMod method [25] proposes to decompose a structure in several subdomains and to build a reduced order model per subdomain. An error indicator allows to find the reduced order models to modify in order to take into account a local modification of the problem. Wang et al. [26] propose a local enhancement of a reduced order model to take into account notches in dynamically loaded panels. A similar approach is used in this paper, without assuming that the local effects induced by the defects are mostly linear. Fluctuation modes are computed on the fly and added in an hyper-reduced order model (HROM). Both meshing and numerical simulation steps are accelerated by the proposed approach.

This work aims at providing a numerical method to assess the mechanical reliability of serial-produced components that can contain voids observed by a non destructive inspection. Since non-parametric defects are considered here, a dedicated data workflow has been developed. The main objective is to assimilate data in order to accelerate forecoming predictions. Fig. 1 provides a simplified workflow of the design and production procedure, respectively denoted "Initial studies" and "Mass production". The durations required for each step are displayed on the time line on the right hand side. The hyperreduced DNS is performed at the quality assessment step if defects are observed during the non destructive inspection. This requires the methodology to be highly adaptable to the input of experimental data. Moreover, results must be provided in a very short time (typically less than a few hours), and be accurate enough to justify the commissioning of the component. The proposed workflow can be compared to the data driven approach proposed in [27]. In the current work, input data are experimental 3D images without any possible parametrization. The data concerning the voids directly come from non destructive inspections, for instance X-ray computed tomography (see Fig. 2]. Even though numerical methods enable the meshing of images [14], this step is not yet sufficiently automated to be applied in an industrial context. As a consequence, methods that can handle non conformal or disconnected meshes are of great interest. Among these methods, one can cite the Arlequin method, that has been coupled with the Latin-PGD method in [28], a discontinuous Galerkin approach for multiscale problems introduced in [29] and the HRM [21]. The present paper focuses on the latter method, described in Section 2.2

The present paper is structured as follows. Section 2 presents the framework of the study. The targeted problem is described and the steps of the methodology are detailed. A first application in 2D is proposed in Section 3 This application is fully parametrized in order to be reproducible. Eventually, Section 4 presents a 3D realistic and non parametric case. 


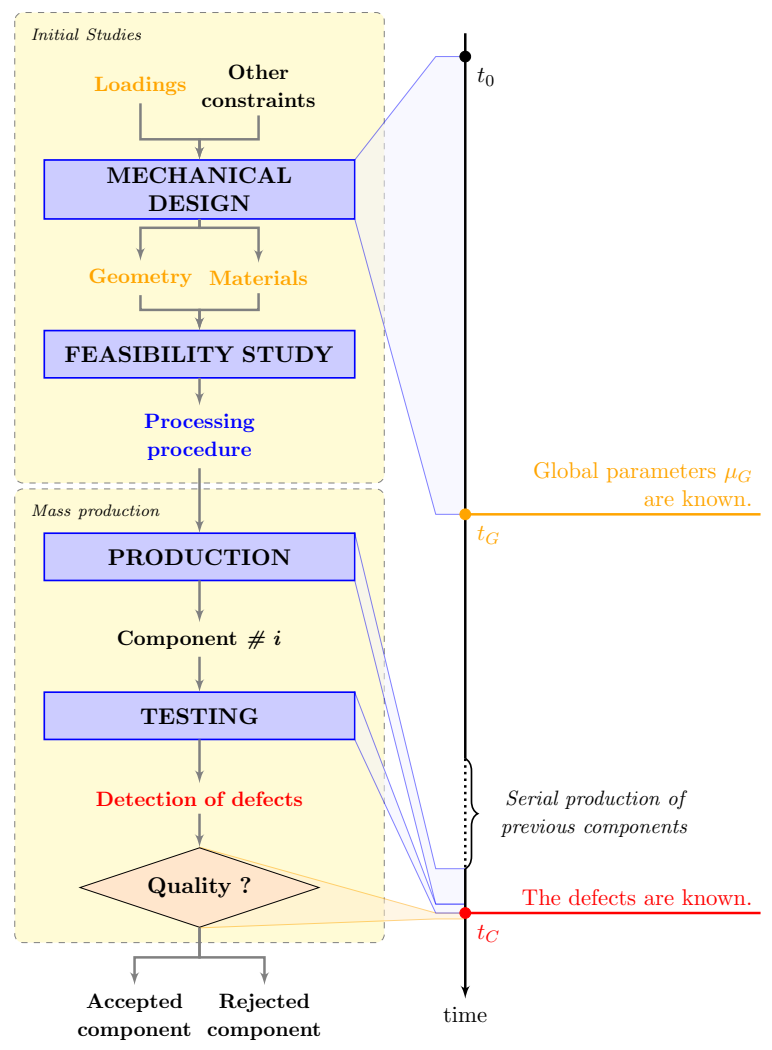

FIGURE 1 Simplified workflow of component design. Some parameters $\left(\mu_{G}\right)$ are known before the mass production, but when defects are observed, an image-based modeling must be performed.

\section{2 | METHOD}

In this section, the targeted mechanical problem is assumed to be non-parametrizable. Thus it does not fit into the classical tensorbased model order reduction framework as presented in Section 2.1 This targeted problem is hence setup like an hyperreduced order model although the related full order model is never simulated (Section 2.2). The reduced basis of this hyperreduced order model is created on the fly once the image of the defect is available. A dedicated workflow is proposed in Sections 2.3, 2.4 \& 2.5 . The case of the defects intersecting a free surface and thus modifying the domain boundary is not adressed here.

\section{1 | Limitations of classical tensor-based model order reduction}

Fatigue criteria are based on stress predictions related to elastoplastic constitutive equations, a weak form of equilibrium equations and cyclic loading. Stresses, denoted by $\sigma$, are the dual variables of a mechanical problem, which primal variables are displacements $\boldsymbol{u}$. Usually, the mechanical problems for displacement and stress prediction are defined over a material domain $\Omega$, a time interval $[0, T]$ and a parameter space $\mathcal{D}_{\mu}$. Formally, the displacement $\boldsymbol{u}(\boldsymbol{x}, t, \boldsymbol{\mu})$ depends on the position $\boldsymbol{x} \in \Omega$, the time $t \in[0, T]$ and the parameters $\mu \in \mathcal{D}_{\mu}$. It is then possible to define a multilinear map from $\Omega \times[0, T] \times \mathcal{D}_{\mu}$ to $\mathbb{R}$ that provides the fields with a tensor structure [30, 31].

Should a classical model order reduction technique based on reduced basis be applied, a low-rank approximation of this tensor is built. In the current study, the material domain $\Omega$ is obtained via computed tomography, or a similar image-based modeling. It is then specific to one particular component. This means that the tensor approximation must be achieved for each component, which is not affordable in a limited time. Moreover the complex shapes (see Fig. 2 of the voids observed cannot be parametrized. The extension of tensor-based model order reduction to direct numerical simulations including variable voids is not straightforward. 


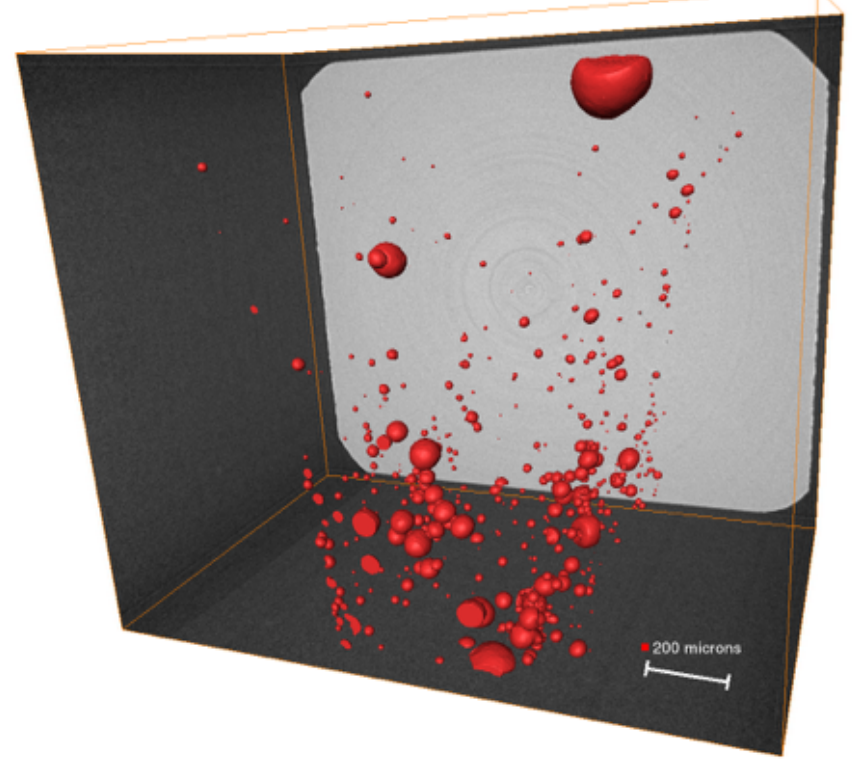

FIGURE 2 3D reconstruction of an X-Ray Computed Tomography image. All volumes in red are voids due to the welding process.

\section{2 | Targeted problem}

This work focuses on solving a mechanical problem involving cyclic elasto-plasticity in a body that contains voids under the small strain assumption. This problem is denoted by $\mathcal{P}^{\star}$. Because of voids, $\mathcal{P}^{\star}$ is component-specific: each produced component contains a unique (possibly empty) set of voids. In the following, the superscript $\star$ refers to mathematical objects that are component-specific. The spatial domain occupied by the studied component is $\Omega^{\star}$ and the time interval of interest is $[0, T]$. The displacement field at point $\boldsymbol{x} \in \Omega^{\star}$ and time $t \in[0, T]$ is denoted $\boldsymbol{u}(\boldsymbol{x}, t)$. The partial differential equation governing this problem is the following, for $t \in(0, T]$ :

$$
\begin{aligned}
\operatorname{div}(\sigma(x, t)) & =0, \quad x \in \Omega^{\star} \\
\sigma(x, t) & =\mathcal{A}(\varepsilon(x, \tau), 0 \leq \tau \leq t), \quad x \in \Omega^{\star} \\
\varepsilon(x, t) & =\frac{1}{2}\left(\nabla u(x, t)+(\nabla u(x, t))^{T}\right), \quad x \in \Omega^{\star} \\
\boldsymbol{u}(\boldsymbol{x}, t) & =u_{0}(\boldsymbol{x}, t), \quad x \in \partial \Omega_{D}^{\star} \\
\boldsymbol{\sigma}(\boldsymbol{x}, t) . \boldsymbol{n} & =T(\boldsymbol{x}, t) . \boldsymbol{n}, \quad \boldsymbol{x} \in \partial \Omega_{N}^{\star}
\end{aligned}
$$

Under the assumption of quasi-static loadings and neglecting the gravity effects, Eq. (1) is the mechanical equilibrium equation. The stress tensor $\sigma$ in the divergence operator of Eq. (1) is obtained through the constitutive equation in Eq. (2). As plasticity is involved, the stress state at a given time $t$ depends on the whole deformation history $\varepsilon(x, \tau), 0<\tau \leq t$. The operator $\mathcal{A}$ is described in the following when the constitutive equations are introduced. As shown in Eq. (3), the deformation is the symmetric part of the displacement gradient. Finally, boundary conditions (BCs) are provided by Eq. (4) and (5). The first one is a Dirichlet boundary condition and imposes displacement values on a part of the boundary of the domain $\partial \Omega_{D}^{\star}$. The BC written in Eq. (5) is a Neumann boundary condition. It imposes the normal stress vector at points of $\partial \Omega_{N}^{\star}$. Only one of these BCs is applied to each point of the domain boundary : $\partial \Omega^{\star}=\partial \Omega_{D}^{\star} \cup \partial \Omega_{N}^{\star}$ and $\partial \Omega_{D}^{\star} \cap \partial \Omega_{N}^{\star}=\emptyset$.

In order to get fast DNS, $\mathcal{P}^{\star}$ is set up as an hyperreduced order model. Solving numerically $\mathcal{P}^{\star}$ aims at estimating the displacement field $\boldsymbol{u}$ over $\Omega^{\star}$ during the time interval $[0, T]$. It is sought as a function of $x \in \Omega^{\star}$ and $t \in[0, T]$, all other parameters being fixed. Under the assumption of separated form of the variables $\boldsymbol{x}$ and $t$, there exist $N^{\star}$ exact modes, denoted by $\left(\boldsymbol{\psi}_{k}^{\star}(\boldsymbol{x})\right)_{k=1, \ldots N^{\star}}$ 
such that:

$$
\begin{aligned}
\boldsymbol{u}(\boldsymbol{x}, t) & =\boldsymbol{u}_{0}(\boldsymbol{x}, t)+\sum_{k=1}^{N^{\star}} \boldsymbol{\psi}_{k}^{\star}(x) \gamma_{k}^{\star}(t) \\
\boldsymbol{\psi}_{k}^{\star}(\boldsymbol{x}) & =\sum_{i=1}^{\mathcal{N}^{\star}} \boldsymbol{\varphi}_{i}(\boldsymbol{x}) \boldsymbol{V}_{i k}^{\star} \quad, \quad k=1, \ldots, N^{\star}, \quad N^{\star} \leq \mathcal{N}^{\star}
\end{aligned}
$$

Here, $\boldsymbol{u}_{0}$ is a given displacement field that fulfills the Dirichlet boundary conditions prescribed over $\partial \Omega_{D}^{\star}$. $\mathcal{N}^{\star}$ is the total number of Degrees Of Freedom (DOFs) in $\mathcal{P}^{\star}$. $\left(\boldsymbol{\varphi}_{i}\right)_{i=1}^{\mathcal{N}^{\star}}$ are the shape functions of the related finite element model. $\boldsymbol{V}^{\star}$ is a $\mathcal{N}^{\star} \times N^{\star}$ matrix containing the nodal components of the exact modes $\left(\boldsymbol{\psi}_{k}^{\star}\right)_{k=1}^{N^{\star}} \cdot\left(\gamma_{k}^{\star}\right)_{k=1}^{N^{\star}}$ are the reduced coordinates in the exact basis. The solution field is then searched in the space spanned by the exact modes. The dimension of this space is generally smaller than the number of DOFs in the problem $\mathcal{P}^{\star}$. In order to set an hyperreduced problem, one still has to determine the Reduced Integration Domain (RID) [20, 32]. This domain denoted by $\Omega_{R}^{\star}$ is a subdomain of $\Omega^{\star}$. This domain is generally made of all the elements containing the interpolation points of the ROBs of interest. Of course the ROB linked to the DOFs of the problem is used, but adding points linked to mechanically relevant ROBs such as stress ROBs improves the hyperreduced prediction [32]. These interpolation points are determined by using the same algorithm as in the Discrete Empirical Interpolation Method [33], a restriction of the Empirical Interpolation Method [34] to orthonormal reduced bases. The set of interpolation indices obtained by applying this algorithm to the displacement modes, respectively the stress modes is denoted $\mathcal{X}^{u}$, respectively $\mathcal{X}^{\sigma}$. Interpolation indices are such that the following restriction, $V^{\star}\left[\mathcal{X}^{u},::\right]$ is an invertible square matrix. A similar property is also obtained for stress modes. The RID is then:

$$
\Omega_{R}^{\star}=\Omega_{u} \cup \Omega_{\sigma} \cup \Omega_{\text {user }}, \quad \Omega_{u}=\cup_{i \in \mathcal{X}^{u}} \operatorname{supp}\left(\varphi_{i}\right), \quad \Omega_{\sigma}=\cup_{i \in \mathcal{X}^{\sigma}} \operatorname{supp}\left(\varphi_{i}^{\sigma}\right)
$$

In Eq. (8), $\Omega_{\text {user }}$ denotes a zone of interest defined by the user. supp is the support of the shape function and $\boldsymbol{\varphi}_{i}^{\sigma}$ are the shape functions related to the stress tensor in $\mathcal{P}^{\star}$ (i.e. the components of symmetric gradient of $\left(\varphi_{k}\right)_{k=1 \ldots \mathcal{N}}$ ). The indices of the DOFS in $\Omega_{R}^{\star}$ that are not at the interface between $\Omega^{\star}$ and $\Omega_{R}^{\star}$ is denoted $\mathcal{F}^{\star}$ :

$$
\mathcal{F}^{\star}=\left\{i \in\left\{1, \ldots \mathcal{N}^{\star}\right\}, \int_{\Omega^{\star} \backslash \Omega_{R}^{\star}}\left(\boldsymbol{\varphi}_{i}^{\star}(\boldsymbol{x})\right)^{2} d x=0\right\}
$$

Solving $\mathcal{P}^{\star}$ using the HRM reads: Find the reduced coordinates $\gamma^{\star}$ that make vanish the projection of the equilibrium residual restrained to $\mathcal{F}^{\star}$ :

$$
\boldsymbol{V}^{\star}\left[\mathcal{F}^{\star},:\right]^{T} \boldsymbol{r}^{\star}\left(\boldsymbol{V}^{\star} \gamma^{\star}\right)\left[\mathcal{F}^{\star}\right]=0
$$

Here, $\boldsymbol{r}\left(\boldsymbol{V}^{\star} \gamma^{\star}\right)$ is the residual of the finite-element equilibrium equations associated to the DOFs values $\boldsymbol{V}^{\star} \gamma^{\star}$. If both $\mathcal{P}^{\star}$ and the related finite element problem $(\boldsymbol{r}(\boldsymbol{q})=0$ ) have a unique solution respectively, then the reduced coordinates computed by solving $\mathcal{P}^{\star}$ are the projections of $\boldsymbol{u}^{\star}-\boldsymbol{u}_{0}^{\star}$ on the exact modes. To have a well-posed problem, the rank of $\boldsymbol{V}^{\star}\left[\mathcal{F}^{\star},:\right]$ must be $N^{\star}$. This is achieved if $V^{\star}$ is a full column rank matrix and if $\Omega_{R}^{\star}$ contains the interpolation points of the exact modes. If the RID is built following the procedure given above, it fulfills this condition. In practice, the larger the RID the more accurate the hyperreduced prediction when using an approximated reduced basis as a substitute to $\boldsymbol{V}^{\star}$. Large RID can be obtained by using the k-SWIM algorithm proposed in [35]. The number of modes $N^{\star}$ is bounded because of the computational complexity of the Newton Raphson algorithm applied to solve Equation 10 . The related linear problem has a full matrix of size $N^{\star}$. But this linear system is sparse in the original finite element problem. The computational complexity of reduced linear solution is proportional to $N^{\star 3}$, compared to a complexity of order $\mathcal{N}^{\star}$ for the finite element problem. In the sequel, we restrict our attention to problems where $N^{\star 3}<\mathcal{N}^{\star}$.

Obviously, the problem $\mathcal{P}^{\star}$ is purely formal, because the exact reduced basis for displacement is unknown. It could be built as soon as all information on the component is available (i.e. at $t_{C}$ on Fig. 1 , but this would require $\Omega^{\star}$ to be meshed and learning simulations to be run. The computational cost and runtime of these two steps are not affordable in a mass production context. In the Hyperreduced DNS, a set of modes is built that approximately spans the same subspace as the exact modes. More precisely, two contributions are taken into account, as illustrated in Fig. 3 The horizontal component of the displacement is plotted on the left of Fig. 3 for a 2D plate containing a hole loaded in the horizontal direction. One can see that the hole has an impact on the plotted field. This displacement field can be written as the sum of the field without the hole $u_{M}$ and a fluctuation field 
$\Delta u_{\mu}$. It is worth noting that the latter field has non-zero values only in the vincinity of the hole. In the following, it is proposed to build two independent ROBs: one for $u_{M}$ and another one for $\Delta u_{\mu}$. This choice is motivated by the fact that it will be exact when the separate scales assumption is met. Then the first contribution is a global one. It describes the mechanical behavior of a defect-free component under loadings of interest. It is assumed that this global contribution can be parametrized by the vector $\boldsymbol{\mu}^{G} \in \mathcal{D}^{G}$ of global parameters (see Fig. 1 . The so-obtained modes are said "idealized" because they are computed on defectfree structures. Finally, when the component is tested at $t_{C}$, the geometry of the problem is fully known. The complexity of the shapes and spatial distribution of the voids does not allow for any parametrization at this stage. The second contribution is a local enhancement, taking into account displacement fluctuations in the vincinity of the voids.

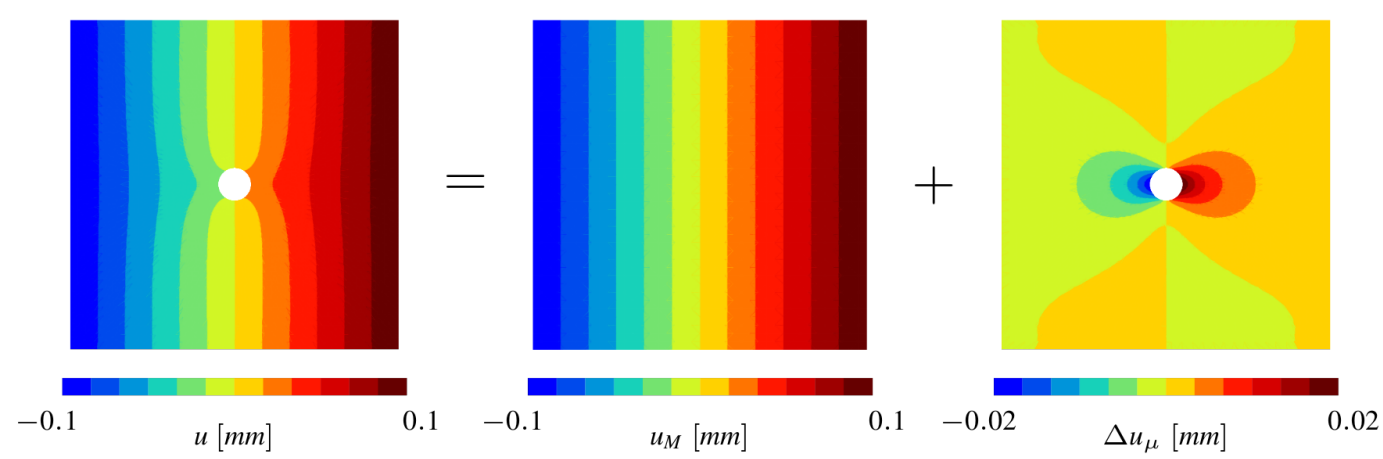

FIGURE 3 Decomposition of the displacement field in a square plate with a hole into a macroscopic component $u_{M}$ (plate without a hole) and a fluctuation part $\Delta u_{\mu}$.

\section{3 | Idealized empirical modes}

Although the voids have no parametric modeling, some features of the targeted problem $\mathcal{P}^{\star}$ can be parametrized. These parameters are related to defect-free models. For instance it contains material or loading parameters, gathered in the vector $\boldsymbol{\mu}^{G}$. One should underline that the information about the component is provided gradually, as shown on the timeline on Fig. 1 . At the end of the mechanical design (at $t_{G}$ ) the global parameter space $\mathcal{D}^{G}$ is defined. At this step, information about the voids is not available. This means that the problems are defined over a domain $\Omega^{G}$ that does not contain defects.

To build the idealized empirical modes, the proper orthogonal decomposition (POD) [18] is applied to the second order tensor $\boldsymbol{u}\left(\boldsymbol{x},\left(t, \boldsymbol{\mu}^{G}\right)\right)$ obtained by grouping $t$ and $\boldsymbol{\mu}^{G}$ in a single multidimensionnal variable. Simulations are run to learn the impact of the global parameters on the mechanical response. Defect-free modes are built by performing a snapshot POD [36] to simulation data generated by defect-free models.

Let $\mathcal{D}_{m}=\left\{\boldsymbol{\mu}_{j}^{G}, j \in 1, \ldots, m\right\}$ be a sampling of $m$ points of $\mathcal{D}^{G}$. The first step aims at extracting the effect of the global parameters. Consequently, $m$ finite element simulations are run. The results are gathered in a matrix of snapshots denoted $\boldsymbol{Q}^{G} \in \mathbb{R}^{\mathcal{N} \times\left(N_{S} \times m\right)}$, with $\boldsymbol{Q}^{G}\left[:, N_{S} \times(j-1)+i\right]=\boldsymbol{u}\left(\boldsymbol{x}, t_{i}, \boldsymbol{\mu}_{j}^{G}\right)$, where $N_{S}$ is the number of snapshots saved per simulation and $t_{i}$ the time at the $i^{\text {th }}$ snapshot. $\mathcal{N}$ is the number of degrees of freedom of the problem. Applying the Singular Value Decomposition to $Q^{G}$ gives :

$$
Q^{G}=V S W^{T}
$$

where $\boldsymbol{V} \in \mathbb{R}^{\mathcal{N} \times \mathcal{N}}$ and $\boldsymbol{W} \in \mathbb{R}^{N_{S} \times N_{S}}$ are orthonormal matrices and $\boldsymbol{S} \in \mathbb{R}^{\mathcal{N} \times N_{S}}$ is a diagonal matrix. The values in $\boldsymbol{S}$ are the singular values of $Q^{G}$ and are decreasing (i.e. if $i \leq j, \lambda_{i} \geq \lambda_{j}$, where $\lambda_{i}=S_{i i}$ ). The POD basis with tolerance $\varepsilon_{t o l}$ is obtained by restraining $\boldsymbol{V}$ to its first $N^{G}$ columns. $N^{G}$ is chosen such that :

$$
N^{G}=\underset{j}{\operatorname{argmin}}\left\{\lambda_{j+1}<\varepsilon_{t o l} \lambda_{1}\right\}
$$


The obtained Reduced Order Basis (ROB) $\boldsymbol{V}^{G} \in \mathbb{R}^{\mathcal{N} \times N^{G}}$ is termed Global Reduced Order Basis (G-ROB). Each column of $\boldsymbol{V}^{G}$ is a global mode. It is defined over the spatial domain $\Omega^{G}$, corresponding to the defect-free component.

\section{4 | Component-specific fluctuation modes}

The quality of an estimation of the fatigue lifetime depends on the accuracy of the prediction of stress and strain fields in zones of interest, while accounting for local plasticity. In the current work, the fatigue crack initiation sites are most likely to be located around the defects. The local mechanical response highly depends on the morphology of the defect and on the applied loading path. To take these two aspects into account, fluctuation modes are built on-the-fly after the inspection of the component.

Let $\Omega^{d}$ be a spatial domain centered on $\boldsymbol{x}_{0}$. A void is introduced whose center of gravity is located at $\boldsymbol{x}_{0}$. The fluctuation induced by this void is defined in Eq (13), where $\boldsymbol{E}(t)$ is the average strain tensor on the domain $\Omega^{d}$ (see Eq. (14)).

$$
\begin{array}{r}
\Delta \boldsymbol{u}(\boldsymbol{x}, t)=\boldsymbol{u}(\boldsymbol{x}, t)-\boldsymbol{E}(t) .\left(\boldsymbol{x}-\boldsymbol{x}_{0}\right) \\
\boldsymbol{E}(t)=\frac{1}{v\left(\Omega^{d}\right)} \int_{\Omega^{d}} \boldsymbol{\varepsilon}(\boldsymbol{x}, t) d v
\end{array}
$$

In the current methodology, $\Omega^{d}$ is chosen such that the dilute assumption is met, i.e. with a very low void volume fraction (typically $10^{-5}$ or $10^{-6}$ ). In Eq. (13), the displacement $u$ is obtained by solving a periodic homogenization problem. The Static Uniform Boundary Condition $\Delta \boldsymbol{u}(\boldsymbol{x}, t)=0, \forall \boldsymbol{x} \in \partial \Omega^{d}, \forall t \in[0, T]$ is used. Because of the low volume fraction, this SUBC has no impact on the fluctuation field in the vincinity of the defect. The loading is applied by imposing the evolution of $\boldsymbol{E}$ over time. We assume that the defect don't modify significantly the effective properties at the scale of the structure. So, this evolution is obtained by extracting the strain path at the defect location from a defect-free simulation. Fluctuation modes $\left(\boldsymbol{\psi}_{k}^{f}\right)_{k=1, \ldots, N_{f}}$ are then built by applying the POD on the snapshot matrix of the fluctuation. They are stored in $\boldsymbol{V}^{f}$. These modes are defined over the fictitious domain $\Omega^{d}$. In the following, they are used to enhance the G-ROB. These modes also have the property to have non zero values only close to the defect :

$$
\forall x \in \partial \Omega^{d}, \forall k \in\left\{1, \ldots, N^{f}\right\}, \psi_{k}^{f}(x)=0
$$

In order to build fluctuations well adapted to the component of interest, these fluctuations modes are built on-the-fly. The strain path at the defect location is extracted from a defect-free calculation. This path is applied as an uniform macroscopic strain $\boldsymbol{E}(t)$ to $\Omega^{d}$. In an homogenization framework, if scale separation between the defect and the structure is not achieved, one has to apply a strain gradient to the matrix. For simplicity reasons, this is not done here. Defects can be very close as shown in Fig. 2 In this case, interactions will take place. If fluctuation modes are built independently for each defect, these interactions will not be well predicted. It is possible to build fluctuation modes for a cluster of closely positioned defects. However, this can fail if one can draw a "path" of close defect along the whole structure.

The fluctuation modes are projected on the component whose spatial domain is $\Omega^{\star}$. It is assumed that the fluctuation modes have a zero value outside $\Omega^{d}$. If the separate scale assumption is met and the defect is far enough from the boundary of this domain, the fluctuation modes take zero values on $\partial \Omega^{\star}$. In this case, the approximation scheme is consistent. However, the methodology presented here has shown good results even if this condition is not fulfilled.

\section{5 | Component-specific calculation}

The component-specific simulation can be set as of $t_{C}$ on Fig. 1 At this time step, the defect population in the welded part is known. The first step of the online procedure is to build the component specific fluctuation modes. The computed fluctuations depend on the loading path applied to the defect, particularly in the case of highly anisotropic defects. To ensure that the obtained fluctuation modes fit the targeted problem, the loading path at the defects' locations is predicted by means of an hyperreduced simulation using only the G-ROB (stored in $\boldsymbol{V}^{G}$ ). Once the fluctuation modes in $\boldsymbol{V}^{f}$ are built, one can run the hyperreduced calculation of the defective part. The idealized empirical modes are defined on the domain $\Omega^{G}$ that does not contain any defect whereas the fluctuation modes are defined over a fictitious domain $\Omega^{d}$ containing the defect.

If a mesh of the defective part $\Omega^{\star}$ is available, the modes are transferred to the new mesh and the RID $\Omega_{R}$ is built by applying the DEIM on the concatenated ROBs. If no mesh is provided, the RIDs can be built independently and merged as follows. Applying the DEIM to $\boldsymbol{V}^{I}$ (respectively $\boldsymbol{V}^{f}$ ) provides a set of magic points in the mesh of $\Omega^{G}$ (respectively $\Omega^{d}$ ). With the elements 
connected to these magic points, one can build the reduced integration domain $\Omega_{R}^{G}$ (respectively $\Omega_{R}^{d}$ ) linked to $\boldsymbol{V}^{G}$ (respectively $\left.V^{f}\right)$. The reduced integration domain for the hyperreduced calculation is $\Omega_{R}=\Omega_{R}^{G} \cup \Omega_{R}^{d}$. If $\Omega_{R}^{G} \cap \Omega_{R}^{d} \neq \emptyset$, one may remove the elements linked to the global scale. Both idealized empirical modes and fluctuation modes are then projected on $\Omega_{R}$. The following matrix $\boldsymbol{V}^{a p p}$ is obtained :

$$
\boldsymbol{V}^{a p p}=\left[\boldsymbol{V}_{R}^{G} \mid \boldsymbol{V}_{R}^{f}\right]
$$

Here, the matrices with a $R$ subscript correspond to the projections on $\Omega_{R}$ of the same matrices defined either on $\Omega^{G}$ or $\Omega^{d}$. $V^{a p p}$ is the Component Specific Reduced Order Basis(CS-ROB). We recall that $N^{\star 3}$ should not be higher than $\mathcal{N}^{\star}$. When this limit is reached, we restrict the modeling to the larger defects such that $N^{\star 3}<\mathcal{N}^{\star}$. Stress modes can also provide additional magic points to build the RID. Section 2.6 gives some details on the procedure to build stress modes at two scales.

Then, it is possible to solve the targeted problem $\mathcal{P}^{\star}$, by replacing $\boldsymbol{V}^{\star}$ to $\boldsymbol{V}^{a p p}, \Omega_{R}^{\star}$ by $\Omega_{R}, \mathcal{F}^{\star}$ by $\mathcal{F}$. Solving $\mathcal{P}^{\star}$ using the HRM now amounts to finding the reduced coordinates $\gamma^{a p p}$ that make the equilibrium residual restrained to $\mathcal{F}$ vanish :

$$
\boldsymbol{V}^{a p p}[\mathcal{F},:]^{T} \boldsymbol{r}^{a p p}\left(\boldsymbol{V}^{a p p} \gamma^{a p p}\right)[\mathcal{F}]=0
$$

Using this method, it is possible to solve the targeted problem with an hyperreduced setting that implies a decreased computational cost. The data workflow with its two contributions to the modes is particularly adapted to the component's life as described in Fig. 1 Indeed, idealized modes at the component scale can be built during the design phase and enhanced on the fly to compute the lifetime of a tested component. It also circumvents the huge offline phase that was needed in 2.2 Moreover, the building of the RID by concatenating meshes suppresses the difficult task of meshing the defective component.

\subsection{Error indicator}

An error indicator for the HROM has been proposed in [37]. It relies on specific stress fields, which are statically admissible to zero (SA0) in a finite element sense. Such a field denoted $\sigma^{S A 0}$ should fulfill the following equations :

$$
\forall i \in\{1, \ldots, \mathcal{N}\}, \quad \int_{\Omega^{\star}} \varepsilon\left(\varphi_{i}^{\star}\right): \sigma^{S A 0} d V=0
$$

Here, $\Omega^{\star}$ is the simulation domain, $\mathcal{N}$ the number of DOFs and $\varphi_{i}^{\star}$ the shape function linked to the $i^{\text {th }}$ DOF. SAO fields can be easlily computed from finite elements computations. If no non zero Neumann BC is applied, the stress fields are already SA0. If some non zero Neumann BC is used, one can run a new simulation with for instance a linear elastic constitutive law. The difference between this field and the previously computed stress field is SA0. As proposed in [37], a ROB of SA0 modes is build and stored in the matrix $\boldsymbol{V}^{\sigma}$. One can then compute the residual of the projection of the current stress state on this ROB:

$$
\boldsymbol{R}(t)=\boldsymbol{q}_{R O M}^{\sigma}(t)-\boldsymbol{V}^{\sigma}\left[\mathcal{F}^{\sigma},:\right] \gamma^{\star}(t), \quad \text { where } \boldsymbol{\gamma}^{\star}(t)=\underset{\gamma}{\operatorname{argmin}}\left\|\boldsymbol{q}_{R O M}^{\sigma}(t)-\boldsymbol{V}^{\sigma}\left[\mathcal{F}^{\sigma},:\right] \gamma\right\|
$$

$\boldsymbol{q}_{R O M}^{\sigma}(t)$ is the vector of the stresses at the integration points of the RID, computed via the constitutive equations. It contains the values of all the stress components at all the Gauss point for time $t . \mathcal{F}^{\sigma}$ is the set of stress components available in $\Omega_{R}$, at Gauss points. This residual is computed on the RID only, that is why $\boldsymbol{V}^{\sigma}[\mathcal{F},:]$ is used in Eq. [19]. A norm of this residual is then integrated over time to produce an error indicator :

$$
\eta_{\sigma}(T)=\sqrt{\frac{\int_{0}^{T}\|\boldsymbol{R}(t)\|^{2} d t}{\int_{0}^{T}\left\|\boldsymbol{q}_{R O M}^{\sigma}(t)\right\|^{2} d t}} \times 100 \quad[\%]
$$

It is worth underlining that this error indicator depends on the stress ROB that is used. As Eq. (19) is restricted to the RID, the error that is computed is a gappy quadrature one. It has been proven in [37] that $\eta_{\sigma}$ is the constitutive relation error when the materials have a linear elastic behavior, if a convenient norm is used in Equation (20) and if the RID contains the whole domain. In the current work, few additionnal steps are followed to build a convenient ROB. It requires the targeted geometry to be meshed in order to compute a reconstruction of a SA0 stress field. This field is the sum of several contributions. The first one is computed on the defect-free structure. The stress field $\sigma^{G}(x, t)$ obtained during the defect-free computations is SA in a FE sense. If Neumann boundary conditions are used in the computation, one has to add a correction field to make it SA0. This correction field can for instance be the opposite of the stress reponse with a linear elastic material. The second contribution takes 
into account the defects by means of a stress fluctuation. This stress fluctuation is defined as follows on the fictitious domain containing the defect $\Omega^{d}$ :

$$
\Delta \sigma(x, t)=\sigma(x, t)-\frac{1}{V\left(\Omega^{d}\right)} \int_{\Omega^{d}} \sigma(x, t) d V
$$

One can then compute the following stress field $\sigma^{R}(x, t)$ :

$$
\boldsymbol{\sigma}^{R}(\boldsymbol{x}, t)=\boldsymbol{\sigma}^{G}(\boldsymbol{x}, t)-\boldsymbol{\sigma}^{\text {Neumann }}(\boldsymbol{x}, t)+\Delta \boldsymbol{\sigma}(\boldsymbol{x}, t)
$$

If the scale separation assumption is met, the stress field $\sigma^{R}$ is SA0 on the domain $\Omega^{\star}$. In the current methodology, this field will be used to provide an error indicator, even if the assumption fails. The snapshot matrix for this field is computed with Eq. (22). A ROB for this stress field is then built as explained previously and used to compute the error indicator $\eta_{\sigma}$. Given a reference full order simulation, one can compute the true error $e_{\sigma}$ :

$$
e_{\sigma}(T)=\sqrt{\frac{\int_{0}^{T}\left\|\boldsymbol{q}_{R O M}^{\sigma}(t)-\boldsymbol{q}_{F O M}^{\sigma}(t)\right\|^{2} d t}{\int_{0}^{T}\left\|\boldsymbol{q}_{F O M}^{\sigma}(t)\right\|^{2} d t}} \times 100 \quad \text { [\%] }
$$

Here, the norms are restricted to the RID. In order to use the error indicator, it needs to be calibrated, i. e. a constant $c_{\eta}$ must be computed so that:

$$
e_{\sigma}(t)=c_{\eta} \times \eta_{\sigma}(t)
$$

To perform this calibration, it is proposed to compute the first time step $t_{1}$ of the structure containing the defects with a full order model and with the hyper-reduced model. The calibration constant is then given by $c_{\eta}=e_{\sigma}\left(t_{1}\right) / \eta_{\sigma}\left(t_{1}\right)$.

\section{3 | IMPACT OF DEFECT SIZE ON THE PERFORMANCE OF THE METHOD}

In this section, the present methodology is applied to investigate the effect of the size of the defects in a welded joint. For sake of reproducibility, a fully parametric case is studied. After a quick review on the simulation set up in Section 3.1 Section 3.2 presents results with different defect sizes, proving that the methodology can be applied even when the scale separation assumption fails. All mechanical calculations are run with the Z-Set software suite [38, 39].

\section{1 | Simulations set up}

\section{Geometry and loading}

The geometry of the weld is idealized as shown on Fig. 4 The plane strain assumption provides a 2D problem. We consider a $20 \mathrm{~mm} \times 2 \mathrm{~mm}$ welded plate. The trapezoidal Fusion Zone (FZ) is at the centre of the plate and has straight sides. Its upper- and lower-width are respectively $2 \mathrm{~mm}$ and $1 \mathrm{~mm}$. In an industrial context, one would like to predict the lifetime of the component to check if the defect will cause an early failure. In the current example, a tensile loading is applied. The normal displacement is set to zero on the left edge of the part. Rigid body motion is fixed by blocking the second displacement on the bottom left node. The loading is applied on the right edge as a prescribed displacement. This loading has a triangular shape with an amplitude of $0.06 \mathrm{~mm}$ and a zero-mean value. No stress is applied to the top and bottom sides of the joint. The number of computed cycles is set to 20. A Newton-Raphson scheme is used to solve the equilibrium at each time step.

\section{Materials}

Two different material behaviors are considered for the Base Metal (BM) and the FZ. The mechanical behavior that is identified for Ti-6Al-4V in [40] has been used for the BM. It relies on von Mises rate-independent plasticity involving a nonlinear isotropic hardening and two nonlinear kinematic hardening variables. The FZ exhibits a martensitic microstructure, whose mechanical behavior has been identified from in-house strain controlled cyclic tests. For the FZ, von Mises plasticity is used along with two 


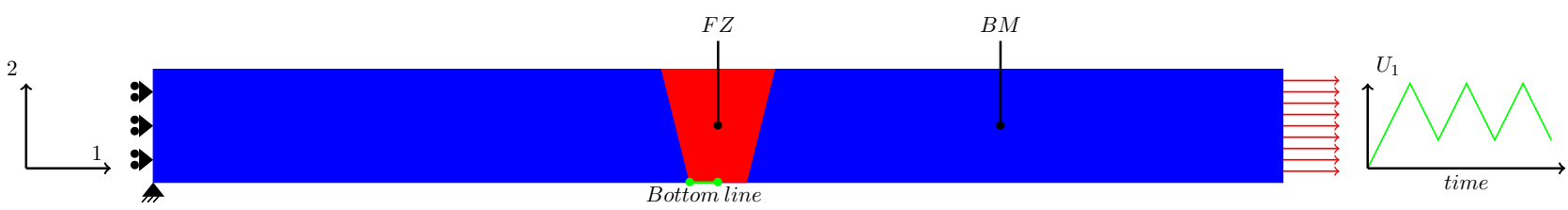

FIGURE 4 Butt joint geometry and loading applied. FZ is Fusion Zone and BM is Base Metal. The Bottom line is a node set of interest to study mesh convergence.

nonlinear kinematic hardening variables and no isotropic hardening. The hardening equations are :

$$
\begin{aligned}
\text { Strain partition } & \varepsilon=\varepsilon^{e}+\varepsilon^{p} \\
\text { Elastic constitutive law } & \sigma=C: \varepsilon^{e} \\
\text { Second invariant of the stress tensor } & J_{2}(\boldsymbol{\sigma})=\sqrt{\frac{3}{2} \operatorname{dev}(\boldsymbol{\sigma}): \operatorname{dev}(\boldsymbol{\sigma})} \\
\text { Yield function } & f\left(\boldsymbol{\sigma}, \boldsymbol{X}_{1}, \boldsymbol{X}_{2}, R\right)=J_{2}\left(\boldsymbol{\sigma}-\boldsymbol{X}_{1}-\boldsymbol{X}_{2}\right)-R \\
& T \\
\text { Cumulated plastic strain } & p=\int_{0}^{T} \sqrt{\frac{2}{3} \dot{\boldsymbol{\varepsilon}}^{p}(t): \dot{\boldsymbol{\varepsilon}}^{p}(t)} d t \\
\text { Kinematic hardening } & \dot{\boldsymbol{X}}_{i}=\frac{2}{3} C_{i} \dot{\boldsymbol{\varepsilon}}^{p}-\gamma_{i} \boldsymbol{X}_{i} \dot{p} \\
\text { Isotropic hardening } & R(p)=R_{0}+Q\left(1-e^{-b p}\right) \\
\text { Kuhn-Tucker conditions } & \dot{p} f=0 ; \dot{p} \geq 0 ; f \leq 0
\end{aligned}
$$

Equations (25) to (32) constitute the operator $\mathcal{A}$ which was defined earlier in Eq.(2). In the following, these equations are integrated with a forward Euler method.

Table 1 summarizes the values of material coefficients. The Poisson ratio of both materials is set to $v=0.32$. In this paper, a non coupled approach is applied to assess the fatigue lifetime of the component [41]. This means that damage is not taken into account in the material's behavior, and that the number of cycles to initiation will be computed by post-processing the stabilized mechanical response [41]. This stabilized response is considered to be reached after 20 cycles. The fatigue life estimation is not presented here. The comparison of the simulations will be made by considering the cyclic stress-strain curves. It is possible to apply the HRM to models with internal length as done in [42] with Cosserat elasticity. This can be useful to regularize damage laws. However, it is worth underlining that, given the ROB building assumptions, the damage will be fairly predicted during the initiation steps. The current procedure will certainly give wrong results once the damage has an impact at the global scale.

TABLE 1 Cyclic behavior coefficients for the different materials.

\begin{tabular}{lcccccccc} 
Material & Young's modulus $(\mathrm{MPa})$ & $R_{0}(\mathrm{MPa})$ & $C_{1}(\mathrm{MPa})$ & $C_{2}(\mathrm{MPa})$ & $\gamma_{1}$ & $\gamma_{2}$ & $\mathrm{Q}(\mathrm{MPa})$ & $\mathrm{b}$ \\
\hline Base Metal & 120350 & 576 & 135000 & 15840 & 750 & 96 & 185 & 71 \\
Fusion Zone & 110000 & 407 & 536000 & 111430 & 1450 & 300 & - & - \\
\hline
\end{tabular}

\section{Meshes}

The meshes are generated using the Gmsh meshing tool [43]. Quadratic triangular elements with reduced integration (4 Gauss points) are used. The mesh of the butt joint is controlled by two parameters: the size of the elements in the FZ and at the edge of the butt joint. The latter is fixed at $0.5 \mathrm{~mm}$. The mesh has been refined until it reaches convergence which is achieved for an element size of $0.025 \mathrm{~mm}$ in the FZ. With this size, both gradient and maximum value of plastic strain do not change when element size is divided by 2 . 


\section{2 | Hyper-reduced simulations with varying defect size}

Simulations have been carried out for four butt joints with circular defects of different sizes as shown in Fig. 5 The position of the defect is held constant in all simulations.

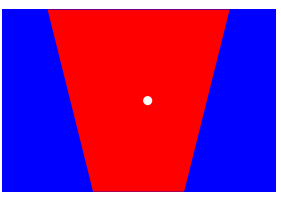

$R=0.05 \mathrm{~mm}$

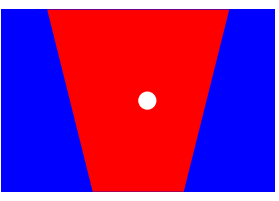

$R=0.1 \mathrm{~mm}$

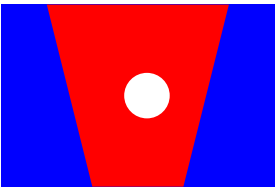

$R=0.25 \mathrm{~mm}$

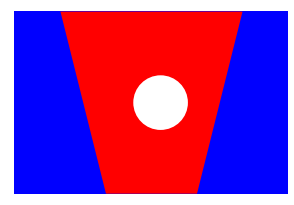

$R=0.3 \mathrm{~mm}$

FIGURE 5 Different configurations of defects considered. $R$ is the radius of the defect. The height of the joint is $2 \mathrm{~mm}$. The defect is located at the half height of the joint and offset by $0.1 \mathrm{~mm}$ to the right.

\section{Offline phase}

In the learning phase, a ROM database that contains the global modes is built. In the following, the global parameters are kept constant equal to $\mu_{0}^{G}$. In this learning simulation, only 1 out of 20 cycles is computed to build the snapshot matrix. Longer cyclic simulations do not improve the accuracy of the idealized modes. The learning simulation is post-processed to build the G-ROB. This ROB contains two modes represented as Modes 1 and 2 on Fig. 6. The first mode presents a quite linear response. The second one underlines the interface between the FZ and the BM. Modes 1 and 2 are computed on a defect free structure and transferred to the mesh containing a defect as represented in Fig. 6 Usual finite element shape functions are used to transfer modes form a mesh to an other.

$U_{1} \quad U_{2}$

Global mode 1

0

Global mode 2

Fluctuation mode 1

Fluctuation mode 2
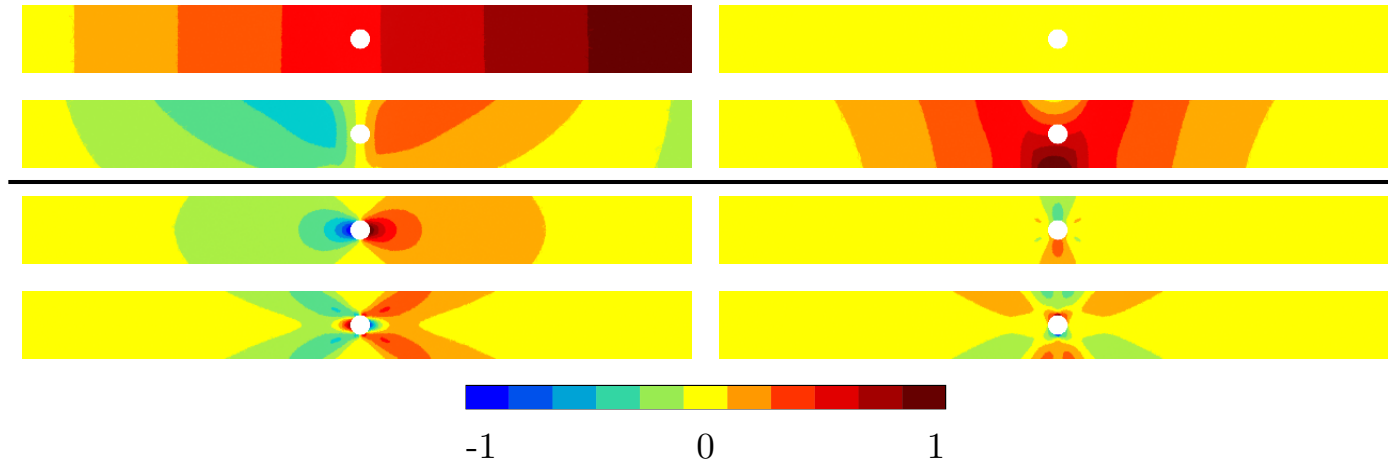

FIGURE 6 Component specific ROB obtained for the biggest defect $(R=0.3 \mathrm{~mm})$. The first two modes are global modes computed on a defect free structure. The last two modes are fluctuations modes computed on a defect embedded in an infinite matrix. One can observe that in this case, the fluctuations computed for the defect have non-zero values on the boundary of the domain.

\section{Fluctuation modes}

The fluctuation modes are built on-the-fly. A first hyperreduced simulation of a defect-free structure is performed. The strain path at the defect location is extracted from this simulation and applied to the defect embedded in an infinite matrix. Fluctuation 
modes are then built by applying the POD on the displacement fluctuations induced by the defect. As the defect position and shape are held constant in this section, the fluctuation modes are built only once and then scaled to fit the size of the considered defect. The obtained modes are shown as Modes 3 and 4 on Fig. 6 . This figure corresponds to the case of the biggest defect. The fluctuation modes are built on a fictitious square domain $\Omega^{d}$. They take a zero value on $\partial \Omega^{d}$, but once scaled and transferred to the structure this is no longer the case. This shows that at least for the biggest defect, the scales are not separate.

Once the modes from the two contributions are concatenated, the RID can be built as explained in Section 2.5. Fig. 7 provides a view of the obtained reduced integration domain for the biggest defect.

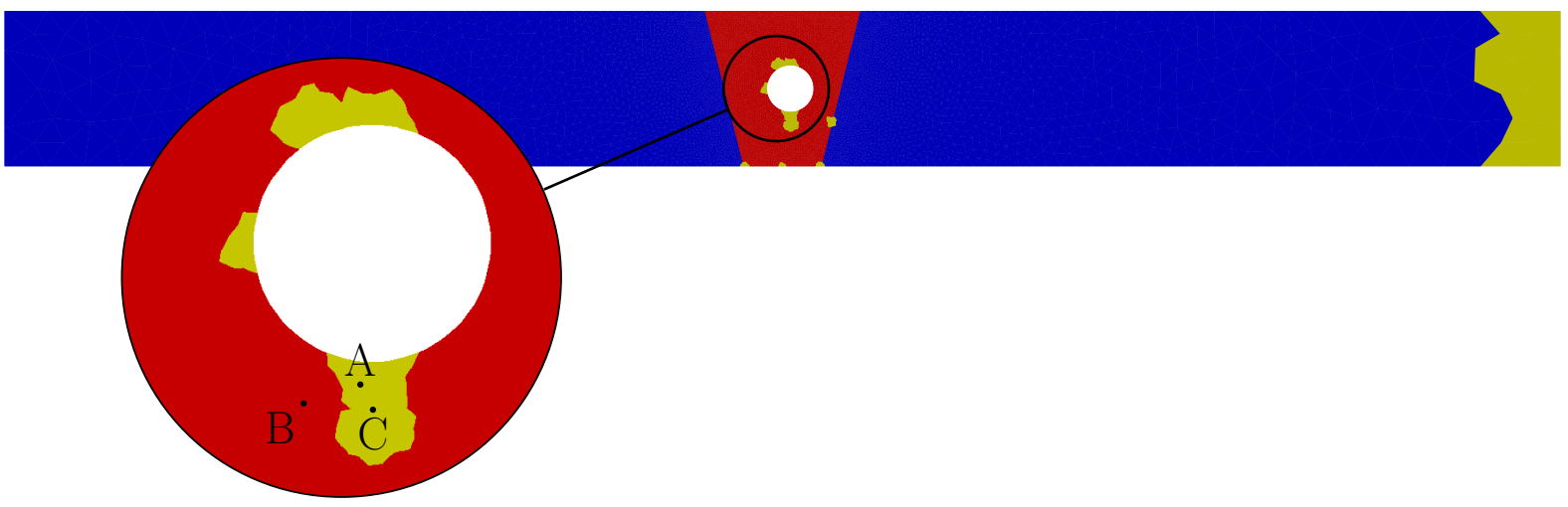

FIGURE 7 Reduced integration domain obtained for the biggest defect. The RID is in yellow, the FZ in red and the BM in blue. The RID contains only 282 of the 14989 elements of the initial mesh. Points labeled A, B and C are used in the following to compare the mechanical response obtained with the HROM to the FOM reference.

\section{Hyperreduced computations}

Twenty cycles are computed with both hyperreduced order model (HROM) and full order model (FOM). FOM is used here as reference for validation. In the current fatigue framework, two quantities of interest (QoI) are studied. The first one is the von Mises equivalent stress $\sigma_{\mathrm{VM}}$ and the second one is the cumulated plastic strain $p$. Relative error indicators are defined in Eq. (33) and (34). In these expressions, the superscript ROM stands for values computed with the hyperreduced order model whereas superscript FOM corresponds to values computed with the full order model.

$$
\begin{gathered}
\xi_{\sigma}(\boldsymbol{x}, t)=\frac{\left|\boldsymbol{\sigma}_{\mathrm{VM}}^{F O M}(\boldsymbol{x}, t)-\sigma_{\mathrm{VM}}^{R O M}(\boldsymbol{x}, t)\right|}{\sigma_{\mathrm{VM}}^{F O M}(\boldsymbol{x}, t)} \times 100 \\
\xi_{p}(\boldsymbol{x}, t)=\frac{\left|p^{F O M}(\boldsymbol{x}, t)-p^{R O M}(\boldsymbol{x}, t)\right|}{\max _{x \in \Omega_{R}} p^{F O M}(\boldsymbol{x}, t)} \times 100
\end{gathered}
$$

Fig. 8 provides a chart to compare the results obtained with the full order model and the reduced order model. Each marker on the chart corresponds to an integration point of the whole domain. The x-coordinate of the marker is the QoI obtained with the FOM, and the y-coordinate is the value obtained with the HROM. The values are computed at the last loading peak. Should the HROM be perfect, all points would lie on the diagonal line drawn in black. If a point is located in the upper-left (resp. lowerright) part of the chart, this means that the HROM overestimates (resp. underestimates) the QoI. Only the case of the largest void $(R=0.3 \mathrm{~mm})$ is shown here. One can observe that the QoIs are well predicted. As the radius of the defect increases, the points are more and more scattered around the diagonal line. For the points that are highly loaded (at the top right of the chart), the prediction remains very satisfying with a tendency of overestimation of the von Mises stress. The maximum relative errors are observed for von Mises stress values around $400 \mathrm{MPa}$, which is close to the yield stress of the material. In this zone, the HROM underestimates the equivalent stress. This means that the predicted plastic zone is slightly smaller.

Fig. 9 displays the stress-stain loops at the last cycle at the points labeled A,B and C on Fig. 7 One can observe that the HROM provides a very good approximation of the local mechanical reponse inside (points A and C). Outside the RID (point B), 

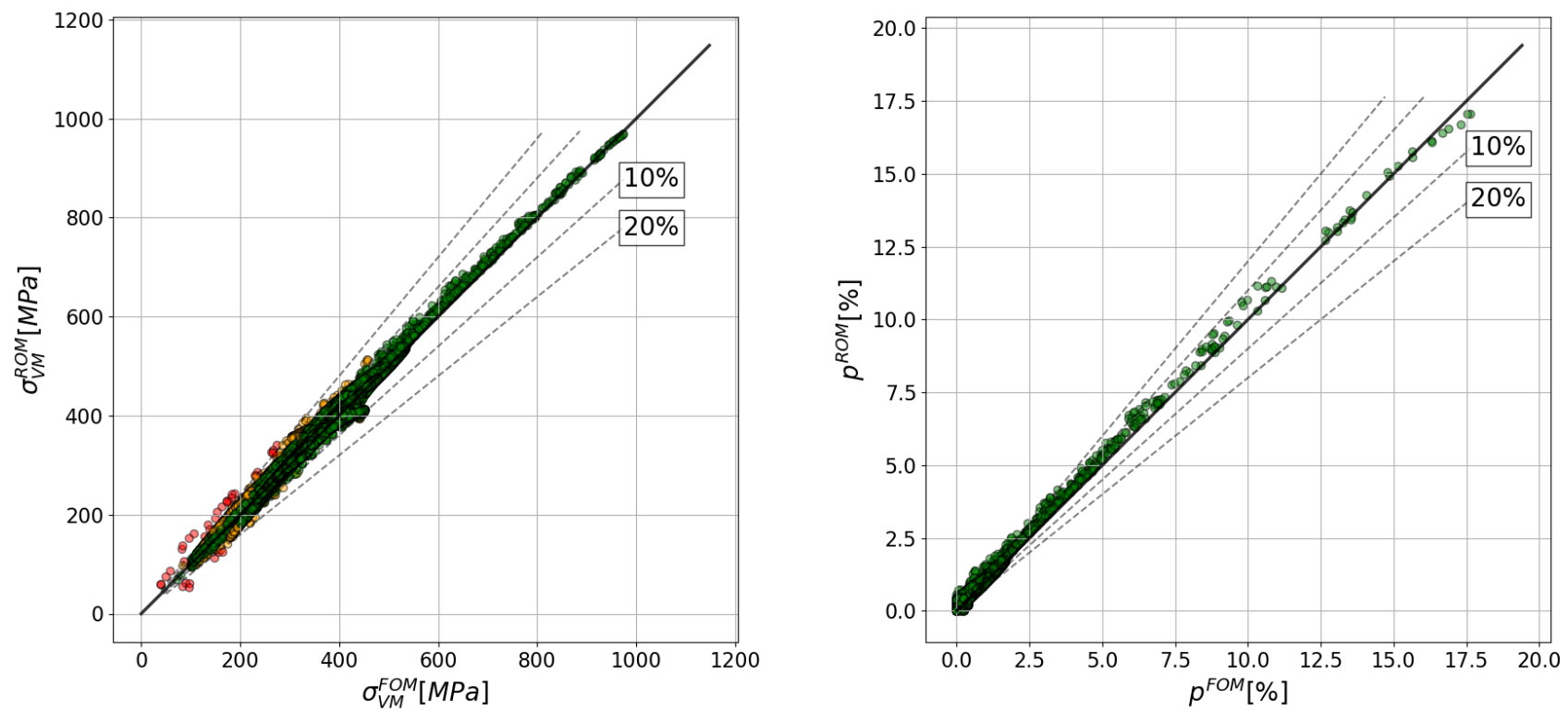

FIGURE 8 Error charts displaying the reference value versus the hyperreduced prediction of the two QoI for each Gauss point of the whole domain at the last loading peak. Only the case of the largest void $(R=0.3 \mathrm{~mm})$ is shown

stress strain loops in the 11 and 22 directions are better predicted than the 12 direction. The results are presented for the biggest defect. In this case, the scales are obviously not separate but the local enhancement by the fluctuation is sufficient to compute a satisfying result. In all other treated cases, the HROM predicts the stress-strain loops very well.

Table 2 summarizes the computational costs of the presented simulations. Two speedups are computed. The first one, termed "No dictionary", considers that the fluctuation modes are not available beforehand. The given value is then the ratio of the CPU time for the full order simulation by the CPU time of the fluctuation modes computation and online phase of the methodology. The second speedup denoted "Dictionary" is computed as if all ROBs were available. It is the CPU time for the full order simulation divided by the CPU time needed for the hyperreduced simulation. The obtained speedups ranging from 30 to 500 are very satisfying given the low error that is observed. The method is more efficient for small defects. Indeed, the smaller the defect the bigger the mesh. When using the Galerkin POD, by choosing $\Omega_{R}=\Omega$, the speedup obtained for the larger defect (case 4 ) is only 4 , when using a dictionary of fluctuation modes. Table 2 also provides some values to quantify the error. $\xi_{\sigma}^{\max }$ is the maximum relative error in von Mises stress at the last peak of loading computed on the RID. $e_{\sigma}$ is the error computed by comparison with the full order simulation as presented in Eq. (23). Finally, $c_{\eta} \eta_{\sigma}$ is the error indicator provided by the HROM, calibrated as explained in Section 2.6 All three indicators increase as the radius of the defect increases. As expected, $\xi_{\sigma}^{\max }$ and $\xi_{p}^{\max }$ which provide a local error are greater than $e_{\sigma}$ and $c_{\eta} \eta_{\sigma}$ that are integrated over the RID. The error indicator of the HROM slightly underestimates $e_{\sigma}$. It is worth noting that the three former indicators require the full order calculation to be computed whereas the latter only needs one time step for calibration.

\section{4 | COMPUTATION ON A REALISTIC 3D WELDED JOINT VIA IMAGE-BASED MODELING}

\section{1 | Position of the problem}

In this section, the presented method is applied to a realistic 3D welded joint, via image-based modeling. The considered geometry is a butt joint with dimensions $20 \mathrm{~mm} \times 1.5 \mathrm{~m} \times 3 \mathrm{~mm}(W \times H \times D)$. The shape of the fusion zone directly comes from a metallographic observation (see [44] for a thourough metallurgical study of this type of welded joints). Fig. 10 provides a view 


\section{Point A}
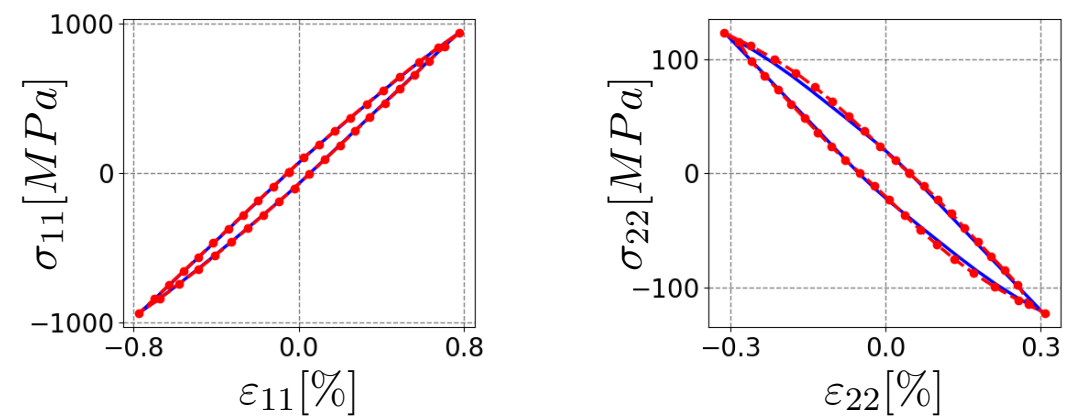

Point B
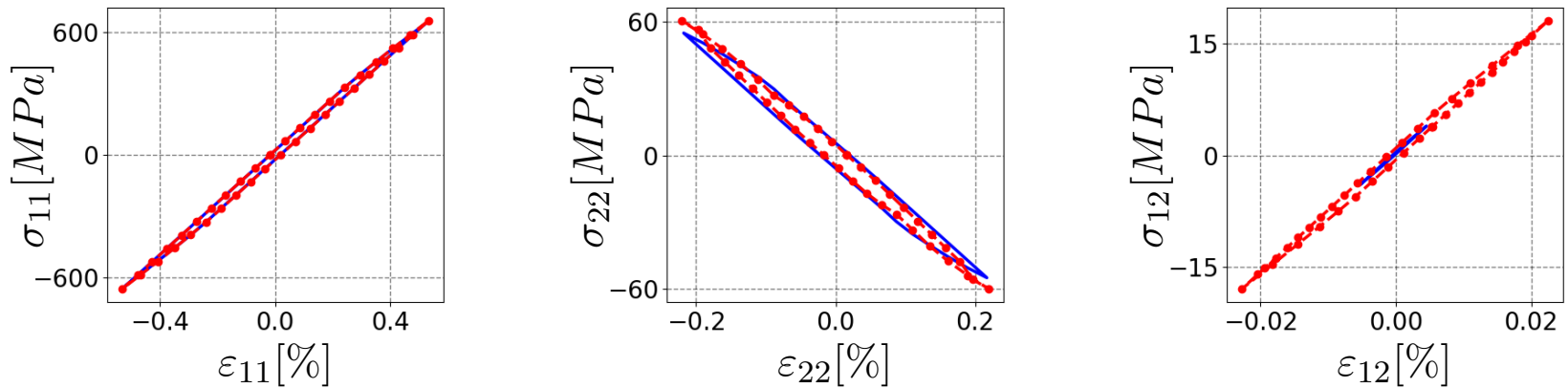

Point C
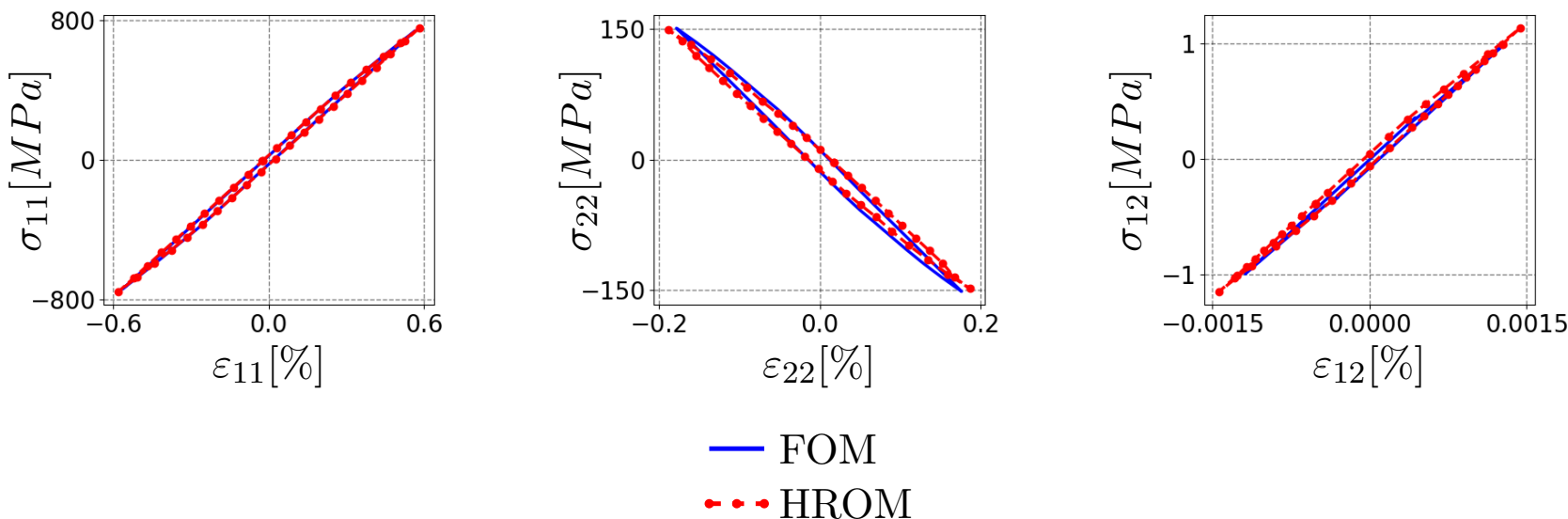

FIGURE 9 Stress-strain loops for points A,B and C shown on Fig. 7

of the geometry. The meshes used in this section are generated with Gmsh [43]. The used elements are quadratic tetrahedra with reduced integration. The full order problem has 795,738 DOFs. A cyclic loading is applied during 50 cycles with a displacement amplitude of $0.1 \mathrm{~mm}$, which corresponds to a macroscopic strain of $\pm 0.5 \%$. The material constitutive laws are the same as in the previous section (see Table 1 . Four defects are introduced in this structure as displayed in Fig. 10 Two defects are spherical and the others directly come from the non destructive inspection of a welded joint: Defect 3 is made of two quasi-spherical parts merged by a small ligament and Defect 4 has a lens shape.

\section{2 | Numerical results}

The G-ROB is obtained by post-processing the simulation of 1 cycle of a defect-free structure (300,000 DOFs). Three modes are obtained. An hyperreduced simulation with the G-ROB only is run to extract the loading path at each defect location. The 
TABLE 2 Tested configurations and CPU times

\begin{tabular}{|c|c|c|c|c|c|c|c|c|c|c|c|}
\hline \multicolumn{2}{|c|}{ Configuration } & \multicolumn{2}{|c|}{ Offline phase } & \multicolumn{2}{|c|}{ Cyclic calculation } & \multicolumn{2}{|c|}{ Speedups } & \multicolumn{4}{|c|}{ Errors [\%] } \\
\hline Case & $\mathrm{R}[\mathrm{mm}]$ & macroscopic & defect & HROM & FOM & No Dictionary & Dictionary & $\xi_{\sigma}^{\max }$ & $\xi_{p}^{\max }$ & $e_{\sigma}$ & $c_{\eta} \eta_{\sigma}$ \\
\hline 1 & 0.05 & $195 \mathrm{~s}$ & $38 \mathrm{~s}$ & $25 \mathrm{~s}$ & $9,480 \mathrm{~s}$ & 150. & 379. & 2.6 & 3.8 & 1.2 & 0.9 \\
\hline 2 & 0.1 & $195 \mathrm{~s}$ & $38 \mathrm{~s}$ & $15 \mathrm{~s}$ & $7,278 \mathrm{~s}$ & 137. & 485. & 2.9 & 5.8 & 2.1 & 1.7 \\
\hline 3 & 0.25 & $195 \mathrm{~s}$ & $38 \mathrm{~s}$ & $26 \mathrm{~s}$ & $2,507 \mathrm{~s}$ & 39. & 96. & 11.2 & 5.8 & 3.9 & 3.8 \\
\hline 4 & 0.3 & $195 \mathrm{~s}$ & $38 \mathrm{~s}$ & $27 \mathrm{~s}$ & $2,197 \mathrm{~s}$ & 33. & 81. & 15.8 & 4.6 & 4.6 & 4.5 \\
\hline
\end{tabular}
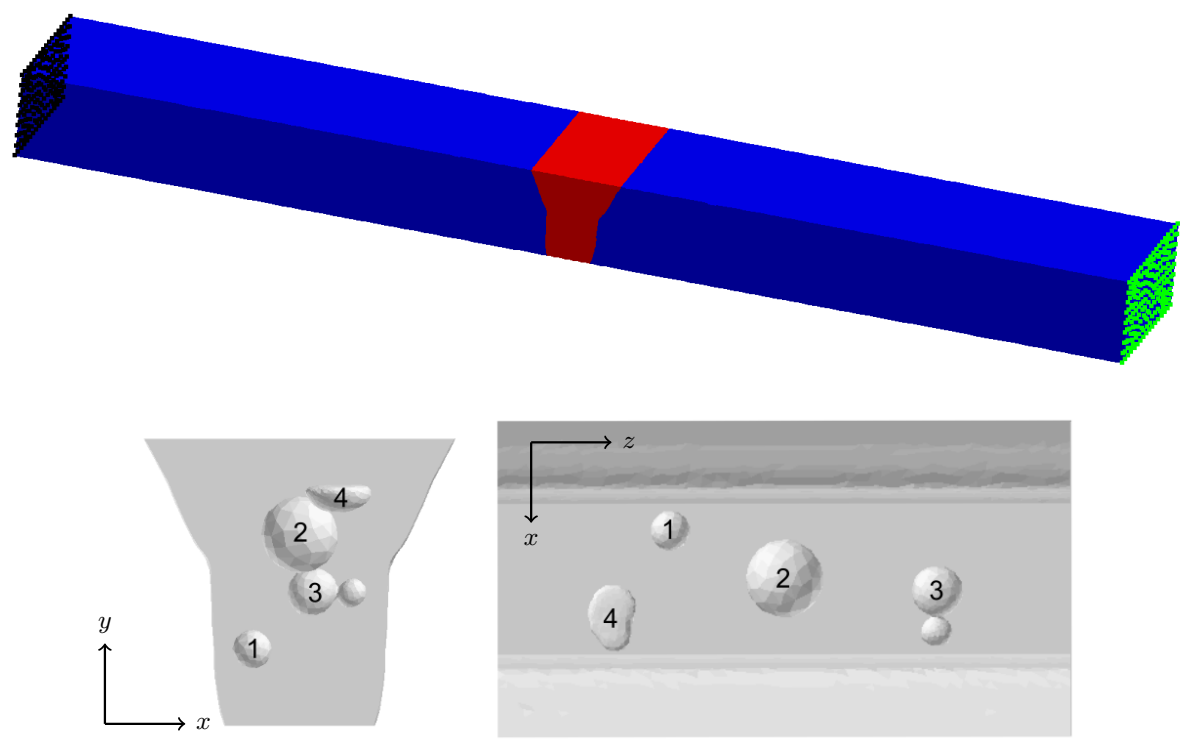

FIGURE 10 View of the considered geometry (top). The FZ is in red, the BM in blue. Loading is applied by imposing a longitudinal displacement on the green nodes. Rigid body motion is fixed by setting to zero the needed displacements on the black nodes. The normal stresses on the four other sides is zero. The defects introduced in the welded joint are viewed from the side (bottom left) and from above (bottom right). Defects 1 and 2 are spherical with respective radii of $100 \mu m$ and $200 \mu m$. Defects 3 and 4 come from a non destructive inspection of welded joint.

fluctuation modes are then built by applying independently this loading paths to the defects embedded in a cubic box. The CSROB is built by concatenating the G-ROB and the fluctuation modes. It contains 15 modes ( 3 from G-ROB +3 fluctuation modes $\times 4$ defects). Fluctuations modes have been computed separately, in parallel, for each defect.

Fig. 11 shows the obtained RID in red. The whole domain is shown in light gray.

Fig. 12 provides a view of the cumulated plastic strain field around the defects. On each face of the skin of the defects is plotted the cumulated plastic strain value at the closest integration point. One can observe that the plasticity is developed in the same zones for both simulations.

Fig. 13 presents the local stress strain loops for the point $C$ which is close to the third defect. The stress-strain loops are well rendered for all components for the directions $11,12,31$. The 31 and 23 shears have very small values. The opening of the 22 loop is well predicted but it overestimates the stresses. The map at the bottom left of the Fig. 13 displays the cumulated plastic strain in the plane of normal $z$ going through the center of gravity of the defect. The second map at the bottom gives the error $\xi_{p}$ on the cumulated plastic strain. This error is under $10 \%$ around the defect.

Fig. 14 presents the error chart obtained for this calculation. Each point represents one integration point at the last loading peak. One can see that the von Mises equivalent stress is well predicted by the HROM. The most loaded points all present a relative error lower than $10 \%$. In this case, the HROM has a tendency to underestimate the von Mises equivalent stress. 


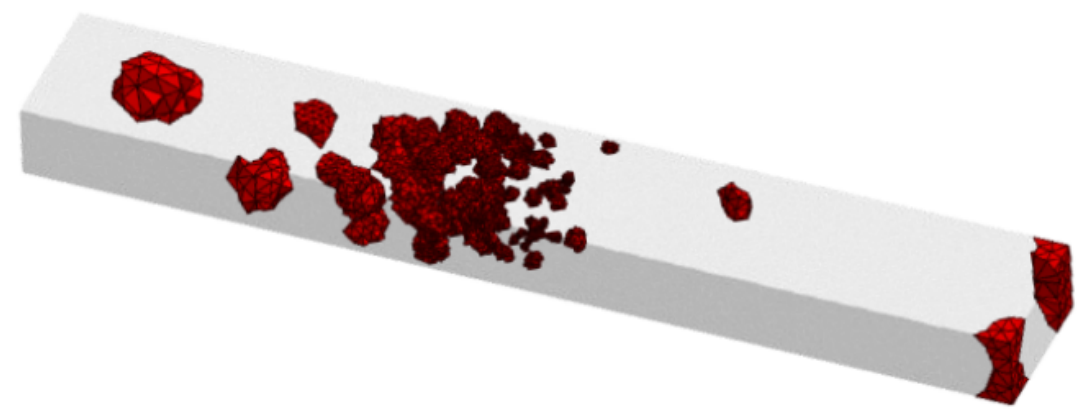

FIGURE 11 View of the Reduced Integration Domain for the 3D case.

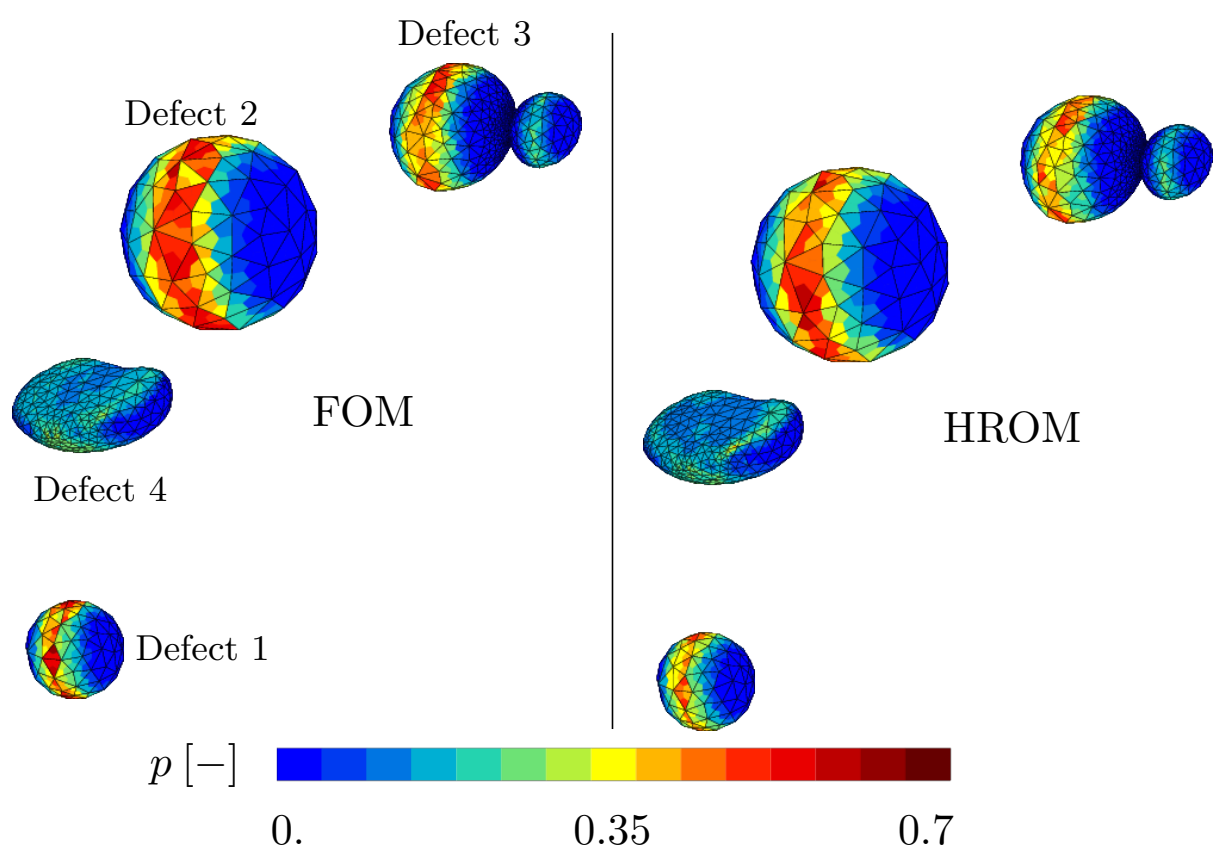

FIGURE 12 Field of cumulated plastic strain $p$ at the end of the simulation for the Full Order Model on the left and the Hyper Reduced DNS on the right.

The error estimation procedure presented in 2.6 has been followed by adding a stress fluctuation per defect. The obtained estimation after calibration is 5.7\%. The true error computed with Eq. 23) is $6.8 \%$. Once again the provided error indicator underestimates the true error.

Two speedups are computed : when considering only the cyclic calculation, the speedup is 1922 . If the building of the fluctuation modes is taken into account for the online time, the speedup is 275 .

\section{5 | CONCLUSIONS}

A combinatorial reduced order modeling by using defect-free structural modes and defect-specific modes related to local strain fluctuations around each defect has been developed. All coupling terms between these modes are taken into account via an hyper-reduced direct numerical simulation. The reduced mesh involved in this model can be built without considering the full 

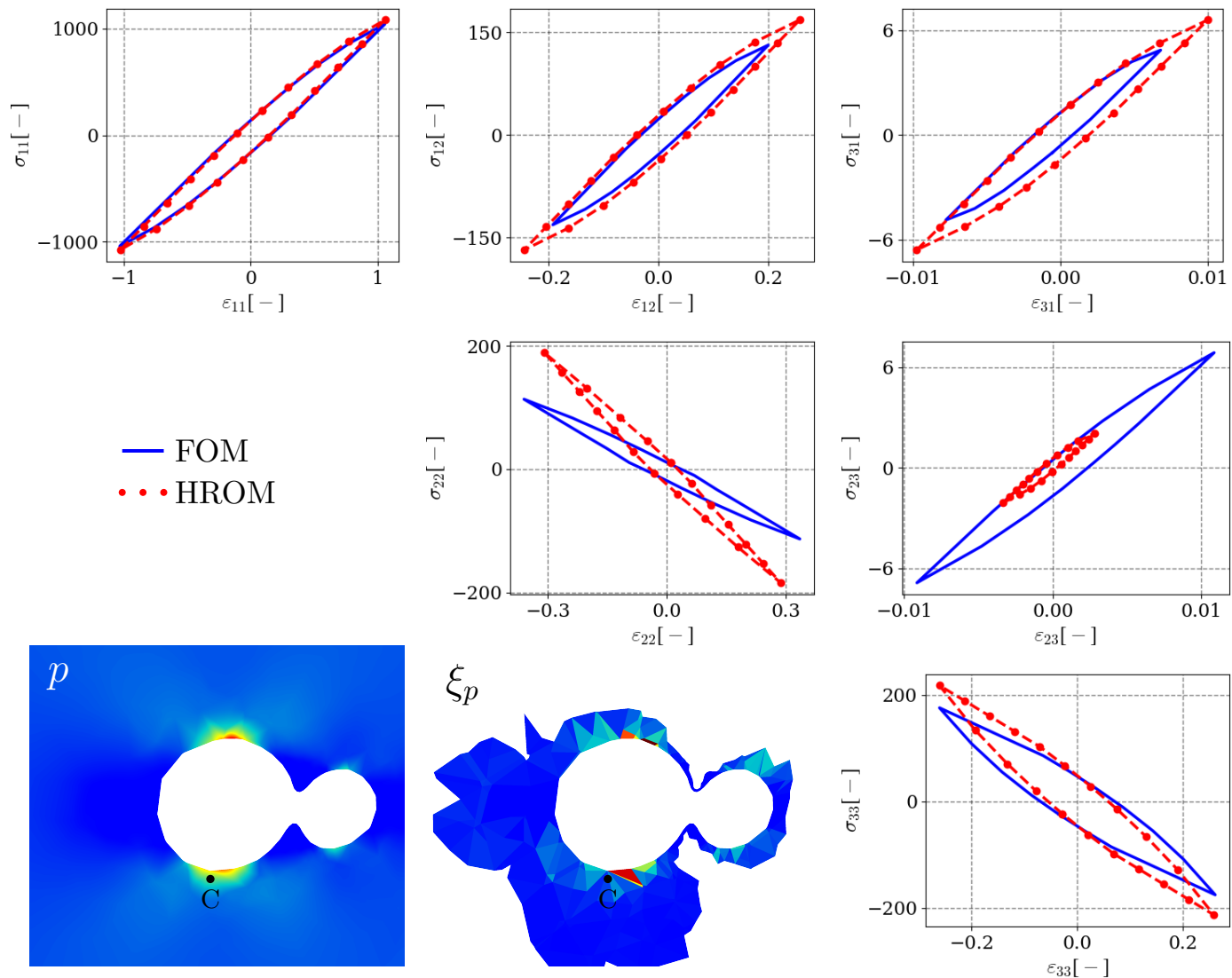

$\xi_{p}[\%] 0$.

4.5

9.

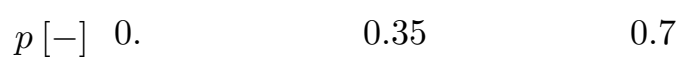

FIGURE 13 Stress strain loops at the point $C$ located close to the third defect. The map on bottom left corner displays the cumulated plastic strain in the plane normal to $z$ going through the center of gravity of the defect 3 . The map at the bottom shows the $\xi_{p}$ error indicator on the RID around the defect 3 .

mesh of the component and its defects. The defect-free structural modes can be computed beforehand. Few modes are computed on the fly to enrich locally the hyper-reduced order model with information from the computed tomography.

The present methodology provides a way of dealing with image-based models that cannot be fully parametrized in a model order reduction framework. The computation of fluctuation modes on the fly enables an enhancement of the ROM to fit a particular problem. Of course, this enhancement is possible using the local fluctuations induced by the defect. This methodology can be extended to other mesoscopic features such as inclusions or small cracks or notches.

From a computational cost point of view, the presented methodology takes advantage of the design calculations to build the global reduced order basis. The fluctuation modes are built out of straightforward simulations of defects in a cubic box. On the whole, the size of the biggest problem to deal with is reduced and this can be interesting in a production context when HPC facilities are not available. The obtained speedups are very satisfying given the error. It is worth noting that the obtained speedups can be improved if the fluctuation modes were previously computed. Indeed, in the online time, building the modes requires more CPU time than running the hyperreduced calculation. One of the main outlooks is to find a way to build a priori the fluctuation modes to improve the speedups. 


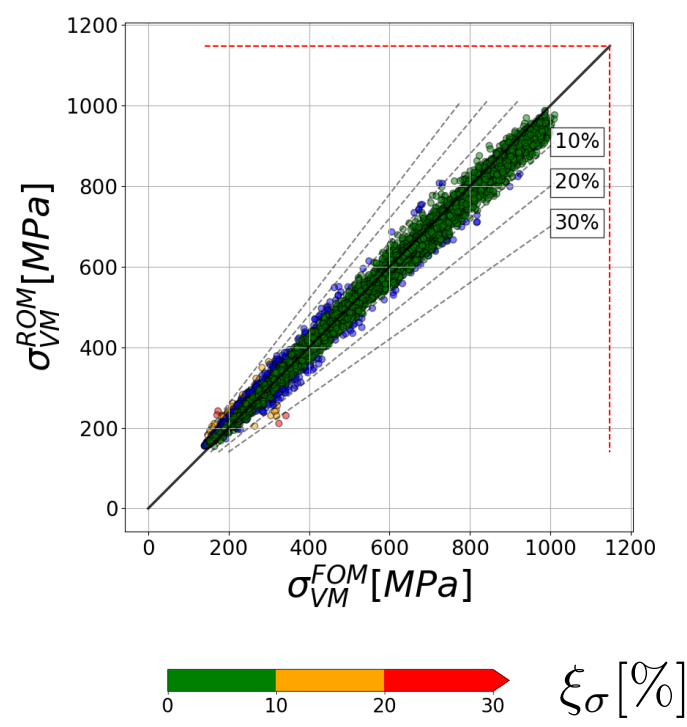

FIGURE 14 Error charts displaying the reference value $\left(\sigma_{V M}^{F O M}\right)$ of the von Mises equivalent stress versus the hyperreduced prediction $\left(\sigma_{V M}^{R O M}\right)$ for each Gauss point of the reduced integration domain at the last peak of loading. The color of the marker is linked to relative error $\xi_{\sigma}$.

\section{References}

[1] Moin P, Mahesh K. Direct numerical simulation: A tool in turbulence research. Annual Review Of Fluid Mechanics. 1998;30:539-578.

[2] Cailletaud G, Forest S, Jeulin D, et al. Some elements of microstructural mechanics. Computational Materials Science. 2003;27(3):351 - 374.

[3] Fish J, Fan R. Mathematical homogenization of nonperiodic heterogeneous media subjected to large deformation transient loading. International Journal for Numerical Methods in Engineering. 2008;76(7):1044-1064.

[4] Fritzen F, Forest S, Bohlke T, Kondo D, Kanit T. Computational homogenization of elasto-plastic porous metals. International Journal of Plasticity. 2012;29:102 - 119 .

[5] Impact of material processing and deformation on cell morphology and mechanical behavior of polyurethane and nickel foams. International Journal of Solids and Structures. 2012;49(19):2714 - 2732.

[6] Chester AS, Bernier JV, Barton NR, Balogh L, Clausen B, Edmiston JK. Direct numerical simulation of deformation twinning in polycrystals. Acta Materialia. 2016;120:348 - 363.

[7] Basseville S, Cailletaud G, Ghidossi T, et al. Numerical analysis on the local mechanical fields in polycrystalline 316LN stainless steel under cyclic fatigue loading: Comparison with experimental results. Materials Science and Engineering: A. 2017;696:122 - 136.

[8] Fomin F, Kashaev N. Influence of Porosity on the High Cycle Fatigue Behaviour of Laser Beam Welded Ti-6Al-4V Butt Joints. In: 3rd International Symposium on Fatigue Design and Materials Defects FDMD 2017; September 19-22 2017; Lecco, Italy.

[9] Mira-Aguiar T, Leitão C, Rodrigues DM. Solid-state resistance seam welding of galvanized steel. The International Journal of Advanced Manufacturing Technology. 2016;86(5):1385-1391.

[10] Uwaba T, Yano Y, Ito M. Resistance spot weldability of 11Cr-ferritic/martensitic steel sheets. Journal of Nuclear Materials. 2012;421(1):132 - 139.

[11] Dinda SK, Warnett JM, Williams MA, Roy GG, Srirangam P. 3D imaging and quantification of porosity in electron beam welded dissimilar steel to Fe-Al alloy joints by X-ray tomography. Materials and Design. 2016;96:224 - 231.

[12] Madison JD, Aagesen L K. Quantitative characterization of porosity in laser welds of stainless steel. Scripta Materialia. 2012;67(9):783 - 786.

[13] Haboudou A, Peyre P, Vannes AB, Peix G. Reduction of porosity content generated during Nd:YAG laser welding of A356 and AA5083 aluminium alloys. Materials Science and Engineering: A. 2003;363(1):40 - 52.

[14] N'Guyen F. Morphologie mathématique appliquée au développement d'outils de maillage EF automatiques dans le cas de microstructures hétérogŔènes bi et multiphasées. In: PhD Thesis, Mécanique Lille 1; 2014. 
[15] Kalidindi SR. Data science and cyberinfrastructure: critical enablers for accelerated development of hierarchical materials. International Materials Reviews. 2015;60(3):150-168

[16] Lorenz EN. Empirical orthogonal functions and statistical weather prediction. MIT Dpt of Meteorology, Statistical Forescasting Project. 1956;Scientific Report 1

[17] Lumley J. The structure of inhomogeneous turbulence. Atmospheric turbulence and wave propagation. 1967;Nauka,Moscow:166-178.

[18] Aubry N, Holmes P, Lumley JL, Stone E. The dynamics of coherent structures in the wall region of a turbulent boundary layer. J. Fluid Mech.. $1988 ; 192: 115-173$

[19] Chinesta F, Ammar A, Leygue A, Keunings R. An overview of the proper generalized decomposition with applications in computational rheology. Journal of Non-Newtonian Fluid Mechanichs. 2011;166(11, SI):578-592. 16th International Workshop on Numerical Methods for Non-Newtonian Flows, Northhampton, MA, JUN 13-16, 2010.

[20] Ryckelynck D. A priori hyperreduction method: an adaptive approach. Journal of Computational Physics. 2005;202(1):346-366.

[21] Fauque J, Ramiere I, Ryckelynck D. Hybrid hyper-reduced modeling for contact mechanics problems. International Journal for Numerical Methods in Engineering. 2018;115(1):117-139.

[22] Phuong Huynh Dinh Bao, Knezevic David J., Patera Anthony T.. A Static condensation Reduced Basis Element method : approximation and a posteriori error estimation. ESAIM: Mathematical Modelling and Numerical Analysis. 2013;47(1):213?251.

[23] Huynh D.B.P., Knezevic D.J., Patera A.T.. A static condensation reduced basis element method: Complex problems. Computer Methods in Applied Mechanics and Engineering. 2013;259:197 - 216.

[24] Ghnatios C, Asmar G, Chakar E, Mosleh C Bou. A reduced-order model manifold technique for automated structural defects judging using the PGD with analytical validation. Comptes Rendus M?canique. 2019;347(2):101 - 113.

[25] Buhr Andreas, Engwer Christian, Ohlberger Mario, Rave Stephan. ArbiLoMod, a Simulation Technique Designed for Arbitrary Local Modifications. SIAM Journal on Scientific Computing. 2015;39:A1435-A1465.

[26] Wang XQ, Phlipot GP, Perez RA, Mignolet MP. Locally enhanced reduced order modeling for the nonlinear geometric response of structures with defects International Journal of Non-Linear Mechanics. 2018;101:1 - 7.

[27] Farhat C, Michopoulos JG, Chang FK, Guibas L J, Lew AJ. Towards a dynamic data driven system for structural and material health monitoring. In: 6th International Conference on Computational Science (ICCS 2006), vol. 3993: :456-464; 2006.

[28] Néron D, Dhia HB, Cottereau R. A decoupled strategy to solve reduced-order multimodel problems in the PGD and Arlequin frameworks. Computational Mechanics. 2016;57(4):509-521.

[29] Kaulmann S, Ohlberger M, Haasdonk B. A new local reduced basis discontinuous Galerkin approach for heterogeneous multiscale problems. Comptes Rendus Mathematique. 2011;349(23):1233 - 1238.

[30] Giraldi L, Nouy A, Legrain G, Cartraud P. Tensor-based methods for numerical homogenization from high-resolution images. Computer Methods in Applied Mechanics and Engineering. 2013;254:154 - 169.

[31] Chinesta F, Ammar A, Cueto E. Recent advances and new challenges in the use of the proper generalized decomposition for solving multidimensional models. Archives of Computational methods in Engineering. 2010;17(4):327-350.

[32] Ryckelynck D, Lampoh K, Quilici S. Hyper-reduced predictions for lifetime assessment of elasto-plastic structures. Meccanica. 2016;51:309-317.

[33] Chaturantabut S, Sorensen DC. Nonlinear model reduction via discrete empirical interpolation. SIAM Journal on Scientific Computing. 2010;32(5):27372764.

[34] Barrault M., Maday Y., Nguyen N. C., Patera A. T.. An Azeempirical interpolationĄf method: application to efficient reduced-basis discretization of partial differential equations. Comptes Rendus Mathématiques. 2004;339(9):667 - 672.

[35] Hith W, Ryckelynck D, Menet C. Data pruning of tomographic data for the calibration of strain localization models. MCA. 2019;0(0):0.

[36] Sirovich L. Turbulence and the dynamics of coherent structures - Part I : Coherent structures. Quarterly of Applied Mathematics. 1987;65(3).

[37] Ryckelynck D, Gallimard L, Jules S. Estimation of the validity domain of hyper-reduction approximations in generalized standard elastoviscoplasticity. Adv. Model. and Simul. in Eng. Sci.. 2015;2:6.

[38] Z-Set User Manual2016.

[39] Besson J, Foerch R. Large scale object-oriented finite element code design. Computer Methods in Applied Mechanics and Engineering. 1997;142(1):165 $-187$.

[40] Naderi M, Hoseini SH, Khonsari MM. Probabilistic simulation of fatigue damage and life scatter of metallic components. International Journal of Plasticity. 2013;43:101-115. 
454 [41] Kruch S, Kanoute P, Bonnand V. Oneras multiaxial and anisothermal lifetime assessment for engine components. AerospaceLab. 2015;.

455 [42] Horák M, Ryckelynck D, Forest S. Hyper-reduction of generalized continua. Computational Mechanics. 2017;59(5):753-778.

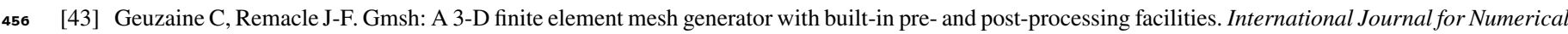
457 Methods in Engineering. 2009;79(11):1309-1331.

458 [44] Sarre B, Flouriot S, Geandier G, Panicaud B, Rancourt V. Mechanical behavior and fracture mechanisms of titanium alloy welded joints made by pulsed 459 laser beam welding. Procedia Structural Integrity. 2016;2:3569 - 3576. 21st European Conference on Fracture, ECF21, 20-24 June 2016, Catania, Italy. 